\title{
CONTROLE DE QUALIDADE ANALÍTICA DOS MÉTODOS UTILIZADOS PARA A DETECÇÃO DE PROTOZOÁRIOS PATOGÊNICOS EM AMOSTRAS DE ÁGUA
}

\author{
R. Cantusio Neto ${ }^{1}$, L.U. dos Santos ${ }^{2}$, M.I.Z. Sato ${ }^{3}$, R.M.B. Franco ${ }^{4}$ \\ 1Sociedade de Abastecimento de Água e Saneamento, Laboratório de Microbiologia, Rua da Abolição, 2375, \\ CEP 13045-750, Campinas, SP, Brasil. E-mail: microbiologia@sanasa.com.br
}

RESUMO

\begin{abstract}
As espécies de protozoários patogênicos de veiculação hídrica Giardia spp.e Cryptosporidium spp. foram responsáveis por cerca $90 \%$ dos surtos de gastroenterite ocorridos nos últimos 25 anos, em vários países. Critérios de avaliação das etapas referentes à detecção destes agentes em amostras de água, tais como: precisão inicial, inoculação nas amostras em água bruta e, diagnóstico e localização de erros estão abordados neste trabalho. Estes critérios devem ser correntemente aplicados e constantemente avaliados pelos laboratórios, a fim de assegurar a confiabilidade dos resultados obtidos durante um monitoramento e, assim, fornecer subsídios para uma avaliação do risco de surtos.
\end{abstract}

PALAVRAS-CHAVE: Controle de qualidade, métodos de detecção, Cryptosporidium spp., Giardia spp., água.

\section{ABSTRACT}

CONTROL OF THE ANALYTICQUALITY OF THE METHODSUSEDFOR THE DETECTION OF PATHOGENIC PROTOZOANS IN WATER SAMPLES. The waterborne pathogenic protozoan species Giardia spp. and Cryptosporidium spp. have been responsible for the majority (90\%) of the gastroenteritis outbreaks that have occurred in the last 25 years in various countries. This paper discusses evaluation criteria pertaining to the steps involved in the detection of these microorganisms in water samples, such as: initial precision, inoculation in environmental samples, diagnosis and error localization. These criteria should be often applied and evaluated by laboratories in order to assure the reliability of the results obtained in a program of monitoring, thus aiding in the evaluation of the risks of outbreaks.

KEY WORDS: Quality assurance, detection methods, Cryptosporidium spp., Giardia spp., water.

A contaminação dos recursos hídricos por protozoários patogênicos de veiculação hídrica tem ocorrido em todo o mundo tornando-se um grande desafio para os sistemas produtores e distribuidores de água. As espécies Giardia spp. e Cryptosporidium spp. foram responsáveis por cerca $90 \%$ dos surtos de gastroenterite ocorridos nos últimos 25 anos em países como os Estados Unidos, Canadá e Japão (KARANIs et al., 2007). No Brasil, a preocupação com estes protozoários patogênicos ao homem e a outros animais levou o Ministério da Saúde a publicar a Portaria 1469, revista sob o n 518/2004; esta publicação recomenda que as Estações de Tratamento de Água pesquisem esses agentes na água a ser distribuída para população, com o objetivo de atingir, como meta, um padrão de ausência. Desta forma, uma devida estruturação laboratorial torna-se necessária. A Agência de Proteção Ambiental Americana (USEPA, 2005) estabeleceu metodologias de detecção de protozoários em amostras de água, baseadas nas etapas de filtração, eluição, concentração, purificação (separação imunomagnética - IMS), coloração e visualização mediante a reação de imunofluorescência direta (RID) realizada com anticorpos monoclonais anti-Cryptosporidium e anti-Giardia já comercializados. Estas metodologias foram internacionalmente validadas, e os laboratórios devem se estruturar com equipamentos e profissionais para atender a esta demanda, sendo necessária também a manutenção da qualidade dos resultados obtidos.

${ }^{2}$ Universidade Estadual de Campinas, Faculdade de Engenharia Civil, Arquitetura e Urbanismo, Departamento de Saneamento e Ambiente, Laboratório de Processos Oxidativos Avançados, Campinas, SP, Brasil.

${ }^{3}$ Companhia de Tecnologia de Saneamento Ambiental, Departamento de Análises Ambientais, São Paulo, SP, Brasil ${ }^{4}$ Universidade Estadual de Campinas, Instituto de Biologia, Departamento de Biologia Animal, Laboratório de Protozoologia, Campinas, SP, Brasil. 
Resultados de ensaios laboratoriais são sempre elementos importantes para a tomada de decisões, pois erros nos testes podem levar a equívocos na condução da solução dos problemas na área ambiental e, consequentemente, em saúde pública (BelLamY, 2004). Programas de qualidade de água podem minimizar erros e aumentar o nível de confiança dos resultados laboratoriais, baseando-se em etapas e adotando métodos para a solução de problemas. Uma proposta de estrutura para o controle de qualidade de metodologias de detecção consiste em estabelecer metas de qualidade que vão desde um gerenciamento dos recursos humanos (promovendo um treinamento atualizado e continuo da equipe de trabalho) até a manutenção física do laboratório e de seus equipamentos (Fig. 1).

O Método 1623 (USEPA, 2005) possui uma Seção de Controle de Qualidade que visa reduzir os possíveis erros e promover dados confiáveis na análise de protozoários de veiculação hídrica como as espécies de Giardia e Cryptosporidium. Desta forma, esse programa inclui a avaliação de manuais de uso, instrumentos e equipamentos usados (calibração), protocolos, qualidade dos reagentes, desempenho e treinamento dos analistas e cartas controle (interno e externo). Neste contexto, a análise mínima requerida nesse programa consisteem demonstrar a capacidade inicial do laboratório em realizar a análise, mediante o teste de precisão inicial e recuperação, de inoculação em matriz (controles positivos), controle negativo (para assegurar ausência de contaminação) e controle de coloração, sendo necessária que esta capacidade seja continuamente demonstrada.

As etapas referentes ao estudo e detecção dos protozoários como: a precisão inicial, a inoculação nas amostras de água bruta e o diagnóstico e localização de erros estão abordados neste trabalho.

\section{Precisão inicial e recuperação}

Por serem realizados com água reagente, os ensaios de precisão inicial atestam a habilidade de execução do método de detecção escolhido, livre de interferências da matriz como, por exemplo, a turbidez da amostra.

A precisão inicial é determinada mediante a contaminação artificial de amostras de água reagente (com um número amostral mínimo de 4), com suspensões contendo um número conhecido de oocistos de Cryptosporidium spp. e cistos de Giardia spp., usualmente com variação entre 100 a 500 organismos; estas amostras são processadas empregando o protocolo adotado pelo laboratório baseado nas etapas de filtração, eluição, concentração, purificação, coloração e visualização de protozoários. Processa-se também uma amostra de água reagente livre de contaminação artificial que é considerada como controle-negativo ecujo resultado atestará as boas condições vigentes no laboratório, pela ausência de contaminação quando processadas várias amostras. Utilizando os resultados gerados por 4 experimentos, são calculados: i) a média da porcentagem de recuperação dos protozoários e ii) o coeficiente de variação (CV, expresso como desvio padrão relativo máximo). Assim, efetuam-se as comparações ao padrão estabelecido pela USEPA: $24 \%$ a $100 \%$ de recuperação para ambos os organismos, Cryptosporidium spp. e Giardia spp. Se a média obtida e o CV estiverem dentro deste critério de aceitação, a performance do sistema é considerada aceitável e as análises de rotina podem ser iniciadas. Caso contrário, ou seja, uma média abaixo do padrão, caracteriza-se desempenho inaceitável do sistema. Neste caso, o problema deve ser corrigido e o teste de precisão inicial deve ser repetidoaté estarem entre os limites aceitáveis (BELLAMY, 2004).

Suspensões purificadas contendo oocistos de Cryptosporidium spp. e cistos de Giardia spp. (inóculos)

Os métodos avaliados para a detecção de Cryptosporidium spp. e Giardia spp. em amostras de água são tecnicamente desafiadores e o desempenho destes é variável entre os laboratórios, entre os analistas e entre os diversos tipos de amostras avaliadas. Essas variações são originadas a partir de pequenas diferenças que ocorrem nos procedimentos e nas propriedades físico-químicas de cada amostra de água (WARNECKE et al., 2003); na marca comercial do kit utilizado no procedimento da etapa de purificação por IMS (no que diz respeito à captura do parasito pelo anticorpo), o que pode afetar a porcentagem de recuperação(REYNOLDsetal.,1999); em fatores biológicos como o tempo de armazenamento dos oocistos ou cistos; a técnica de purificação dos organismos e a etapa de inoculação artificial nas amostras de água. Todos estes fatores podem desempenhar um importante papel na recuperação dos organismos (Hsu; YeH, 2003).

Em relação às suspensões vendidas comercialmente existem: a) o inóculo Easy Seed ${ }^{\circledR}$ TM BTF (Biotechnology Frontiers; Austrália)éuma "suspensão teste" de cistos e oocistos contidos em um tubo de 5 $\mathrm{mL}$, com número conhecido (100 oocistos e 100 cistos $\pm 1 \mathrm{DP})$ preservados em, aproximadamente, $1 \mathrm{~mL}$ de solução salina. A citometria de fluxo é usada para enumerar estas formas e o desvio padrão do número deoocistos ecistos decada tuboé menor que 2,5. Cada tubo é selado e esterilizado expondo cada lote a uma dose de radiação gama, garantido a estabilidade e inativação dos organismos. Énormalmenteusadopara contaminar artificialmenteas amostras em avalia-ções iniciais de precisão dos métodos. 


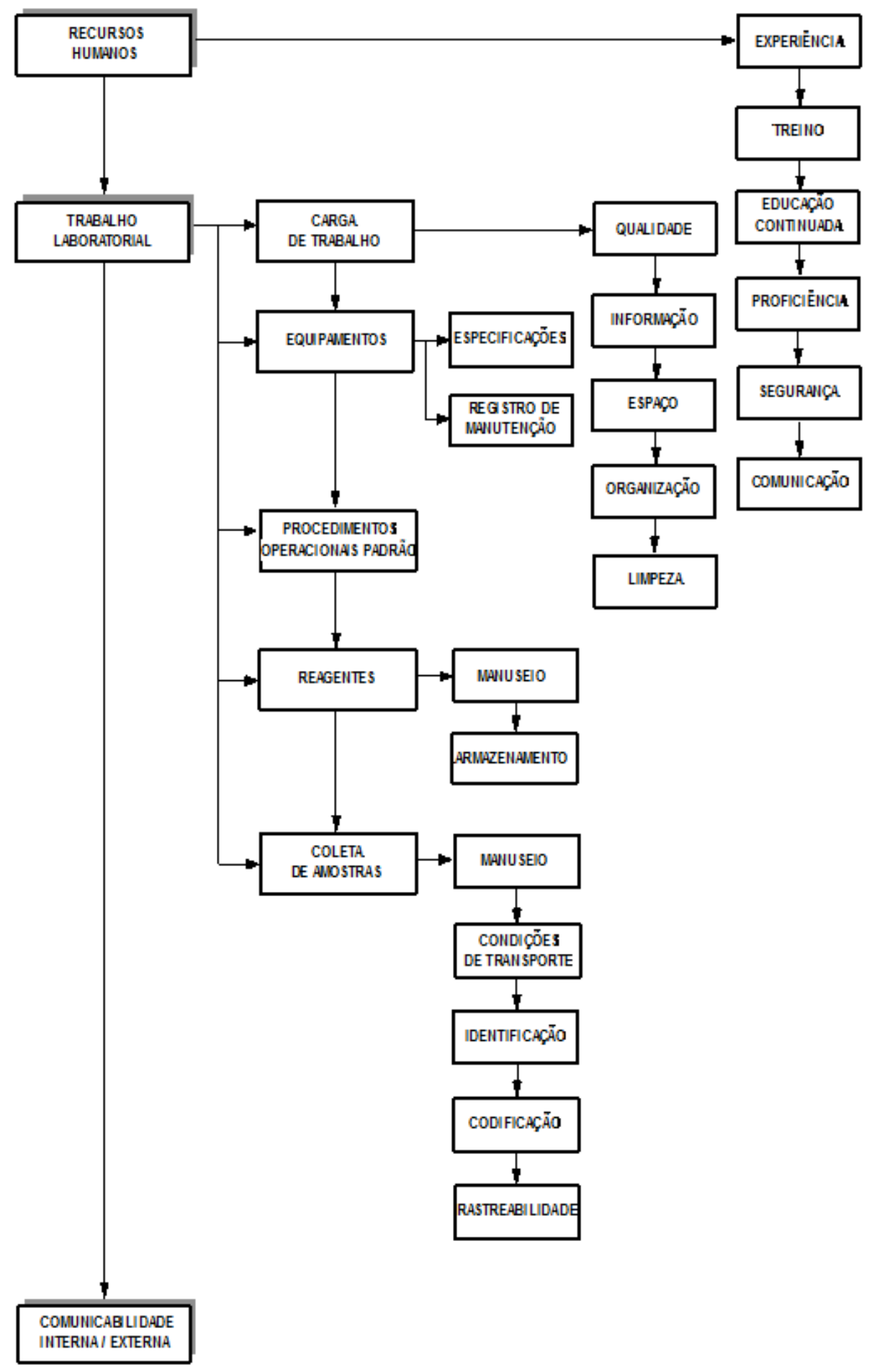

Fig.1 - Programa de qualidade operacional.

O inóculo Color Seed ${ }^{\circledR}$ é uma suspensão de oocistos e cistos marcados com corante vermelho fluorescente, ou seja, a sulfo-rodamina, que constitui o corante "Texas Red" (WARNECKE et al., 2003), contidos em um frasco de $5 \mathrm{~mL}$, com número aproximado de 100 oocistos e 100 cistos $\pm 1 \mathrm{DP}$, também preservados em aproximadamente $1 \mathrm{~mL}$ de solução salina, e enumerados por citometria de fluxo, o mesmo desvio padrão do número dos organismos em cada tubo menor que 2,5 e, inativados com uma dose de radiação gama. Desta forma, por serem marcados com outro fluorocromo, são usados como "autocontrole interno" em amostras naturais, pelo fato da leitura microscópica ser realizada com filtros 
de diferentes comprimentos de onda, em relação ao fluorocromo isotiocianato de fluoresceína (FITC). Com a mudança do conjunto de filtros, é realizada a visualização dos organismos naturalmente presentes na amostras (observados na cor verde maçã brilhante - FITC) e os organismos usados na contaminação artificial (observados em vermelho), ou seja, se o organismo fluoresce em vermelho, ele é contado como o controle-positivo (Color Seed ${ }^{\circledR}$ ); porém, se há fluorescência em verde, écontado como intrínseco da amostra (contaminação natural).

Esteinóculo apresenta a característica de produzir uma pequena queda na eficiência de recuperação para cistos e oocistos (WARNECKE et al., 2003) sendo que isto se deve, provavelmente, ao aumento da hidrofobicidade dos organismos causada pela adesão aos marcadores de hidrocarboneto (WARNECKE et al., 2003), resultando no aumento da aderência nas superfícies, além de perdas durante os processos de concentração e análise. Embora seu uso seja considerado estável para uma ampla variação de condições, ao ser comparado com uma suspensão não comercial de cistos e oocistos, o Color Seed ${ }^{\circledR}$ apresentou uma recuperação cerca de 4,0 \% menor de organismos (WARNECKE et al., 2003).

Considera-se que a obtenção de resultados variáveis de recuperação seja inerente aos diferentes métodos de deteç̧ão, como também a baixa reprodutibilidade, pois um mesmo laboratório alcança valores distintos em diferentes ensaios (FRICKER; CRAB, 1998; CooK et al., 2006).

Sabe-se que a produção de inóculo é desafiadora. A enumeração das formas (cistos e oocistos) é uma etapa crítica, uma vez que os métodos de contagem não são totalmente eficientes e o problema da distribuição não homogênea dos organismos na suspensão, que pode levar à não exatidão do número de cistos e oocistos inoculados, gera uma grande variabilidade nos valores de recuperação (FERGUSON et al., 2004). Ainda, fatores como aspectos biológicos (idade e origem dos organismos), procedimentos prévios de limpeza e meio de armazenamento tambéminfluenciam nos valores derecuperação (BUKHARI et al., 1998; LINDQUIST et al., 1999).

\section{Inoculação em matriz de água bruta}

Nesta etapa, procede-se à contaminação artificial de uma alíquota da amostra de água bruta (amostras de campo) com uma quantidade conhecida de oocistos de Cryptosporidium spp. e de cistos de Giardia spp. marcados com sulfo-rodamina. Esta etapa visa avaliar o efeito da matriz (amostras de água natural ou bruta) sobre a recuperação dos mesmos (com base na contaminação artificial com inóculo Color Seed $\left.{ }^{\circledR}\right)$, e deve ocorrer a cada 20 amostras de campo analisadas. As amostras, inoculadas ou aquelas referentes ao controle-negativo, devem ser processadas de acordo com o protocolo de processamento adotado pelo laboratório.

As recuperações obtidas nesta etapa devem estar dentro dos limites estabelecidos pela USEPA que são: $13-111 \%$ para Cryptosporidium spp. e $15-118 \%$ para Giardia spp. Da mesma forma comona precisão inicial, após análise dos experimentos inoculados, são feitos os cálculos da média de porcentagem de recuperação e do desvio padrão relativo. A precisão aceita pode ser expressa através do intervalo obtido da média de recuperação, mais ou menos dois desvios-padrão.

\section{Deteç̧ão decistos e oocistos: etapa de visualização}

O equipamento de microscopia utilizado para visualização depende de alinhamento e ajuste das várias partes ópticas, que são sofisticadas. Sem estes ajustes, o microscópio de fluorescência não funcionará com sua eficiência máxima, dificultando a identificação e enumeração dos organismos. Ajustes no feixe de luz da lâmpada de mercúrio da epifluorescência são necessários para resultar na regular iluminação do campo. Este ajuste deve ser realizado antes do primeiro uso do microscópio, na troca de lâmpadas ou quando problemas com a fluorescência ocorrerem. A calibração da ocular micrométrica, utilizada para medir o tamanho dos organismos é outro exemplo de controle que devem estar devidamente registrados no Manual de Qualidade Analítica (USEPA, 2005).

O desempenho do microscopista deve também ser constantemente avaliado realizando-se periodicamente a visualização de lâminas coradas com o corante vital DAPI (positivo e negativo), usado como teste confirmatório de morfologia, observando-se as características morfológicas dos organismos adotados como critério. A análise destes critérios deve manter-se em torno de 10,0 \% entre os microscopistas. Laboratórios que contam apenas com um microscopista devem manter fotografias de oocistos e cistos e dos organismos interferentes, para comparação dos resultados, realizarem várias observações da mesma lâmina ou, ainda, fazerem parcerias com outros laboratórios para uma comparação interlaboratorial dos resultados (USEPA, 2005).

\section{Diagnóstico e localização de erros}

Se os resultados obtidos nas etapas acima descritas estiverem fora dos limites estabelecidos e a causa não for determinada, alguns procedimentos analíticos podem colaborar para uma melhora na performance dos procedimentos adotados, tais como:

a) checagem do sistema de microscopia e o anticorpo analisado, verificando a iluminação do microscópio e examinando alíquotas da suspensão controle posi- 
tivo presentes nos kits comerciais dos anticorpos, observando que mais de $50 \%$ dos oocistos e cistos apresentem fluorescência compatível com os padrões previamente estabelecidos;

b) checagem da etapa de purificação pela IMS por meio da inoculação de 100 a 500 organismos em $10 \mathrm{~mL}$ de água reagente e processamento desta amostra com a separação imunomagnética seguida da visualização;

c) checagem das etapas de filtração, concentração e eluição, através da contaminação artificial em água reagente e execução dessas etapas seguidas da visualização, observando-se a integridade de oocistos e cistos durante a leitura das lâminas.

Estes critérios de avaliação devem ser correntemente aplicados e constantemente avaliados pelos laboratórios (CLANCY et al., 1999; LindQUist et al., 1999) a fim de assegurar a confiabilidade dos resultados de detecção, pois a importância do conhecimento de parâmetros parasitológicos dos mananciais, obtidos durante um monitoramento, pode fornecer subsídios para uma avaliação de risco e minimizar a possibilidade de surtos. Destaca-se como exemplo o grande surto de Milwaukee que, além de causar 100 mortes, gerou um gasto de U\$ 96,2 milhões aos cofres públicos americano, sendo U\$ 31,7 milhões referentes aos custos médicos e 2 vezes mais (cerca de U\$ 64,6 milhões) em perda de produtividade (SunNotel et al., 2006; FAYER, 2004). Pesquisadores, ao avaliarem os modelos de risco da criptosporidiose, concluíram que mediante o monitoramento obtém-se uma melhor avaliação da possibilidade de ocorrência da doença em níveis endêmicos e a determinação das fontes e causas dos surtos; informações estas que poderão ser usadas para minimizar sua transmissão através da água (ROSE et al., 2002) visando o controle desta protozoose.

O conhecimento obtido também contribui para o delineamento de programas visando à recuperação dos recursos hídricos, sendo necessário, portanto, que os resultados gerados no monitoramento sejam os mais confiáveis possíveis.

\section{REFERÊNCIAS}

BELLAMY, J.E.C. Quality assurance considerations for detection of waterborne zoonotic parasites using Cryptosporidium oocyst detection as the main example. Veterinary Parasitology, v.126, p.235-248, 2004.

BRASIL. Ministério da Saúde. Portaria 1469, de 29 de dezembro de 2000. (Republicada no D.O. n¹ de de 02/01/2001); Brasília. 2000.

BRASIL. Ministério da Saúde. Portaria 518, de 24 de março de 2004. Padrão de Potabilidade da Água para Consumo Humano. Brasília, 2004.
BUKHARI, Z.;McCUIN, R.M.; FRICKER, R.C.; CLANCY, J.L. Immunomagnetic separation of Cryptosporidium parvum from source water samples of various turbidities. Applied Environmental Microbiology, v.64, p.4495-4499, 1998.

CLANCY, J.L.; BUKHARI, Z.; McCUIN, R.M.; MATHESON, Z.; FRICKER, C.R. USEPA

Method 1622. Journal of the American Water Works Association, v.91, p.60-67, 1999.

COOK, N.; PATON, C.A.; WILKINSON, N.; NICHOLS, R.A.B.; BARKER, K.; SMITH, H.V. Towards standard methods for the detection of Cryptosporidium paroum on lettuce and raspberries. Part 1: Development and optimization of methods. International Journal of Food Microbiology, v.109, p.215-221, 2006.

FAYER, R. Cryptosporidium: a water-borne zoonotic parasite. Veterinary Parasitology, v.126, p.37-56, 2004.

FERGUSON, C.; KAUCNER, C.; KROGH, M.; DEERE, D.; WARNECKE, M. Comparison of methods for the concentration of Cryptosporidium oocysts and Giardia cysts from raw waters. Canadian Journal of Microbiology, v.50, p.675-682, 2004.

FRICKER, C.R.; CRABB, J. Waterborne cryptosporidiosis: detection methods and treatment options. Advances in Parasitology, v.40, p.241-278, 1998.

HSU, B.M.; YEH, H.H. Removal of Giardia and Cryptosporidium in drinking water treatment a pilot-scale study. Water Research, v. 35, p. 1111-1117, 2003.

KARANIS, P.; KOURENTI, C.; SMITH, H. Waterborne transmission of protozoan parasites: A worldwide review of outbreaks and lessons learnt. Journal of Water and Health, v.5, n.1, p.1-38, 2007.

LINQUIST, A.; DUFOUR, A.P.; WYMER, L.J.; SCHAEFER III, F.W. Criteria for evaluation of proposed protozoan detection methods. Journal of Microbiology Methods, v.37, p.33-43, 1999.

REYNOLDS, D.T.; SLADE, R.B.; SYKES, N.J.; JONAS, A. and FRICKER, C.R. Detection of Cryptosporidium oocysts in water: techniques for generating precise recovery data. Journal of Applied Microbiology, v.87, p.804-813, 1999.

ROSE, J.B.; HUFFMAN, D.E.; GENNACCARO, A. Risk and control of waterborne cryptosporidiosis. FEMS Microbiology Reviews, v.26, p.113-123, 2002.

SUNNOTEL, O.; LOWERY, C.J.; MOORE, J.E.; DOOLEY, J.S.G.; XIAO, L.; MILLAR, B.C.; ROONEY, P.J.; SNELLING, W.J. Cryptosporidium. Letters in Applied Microbiology, v.43, p.7-16, 2006.

UNITED STATES ENVIRONMENTAL PROTECTION AGENCY (USEPA). Office of Water. Washington DC. 
Method 1623: Cryptosporidium and Giardia in Water by filtration/IMS/FA. EPA-815-R-05-002, 2005.

WARNECKE, M.; WEIR, C.; VESEY, G. Evaluation of an internal positive control for Cryptosporidium and Giardia testing in water samples. Letters in Applied Microbiology, v.37, p.244-248, 2003.

Recebido em 1/6/2/09

Aceito em 5/8/10 